\title{
Predisposing factors for multi drug resistant Tuberculosis in Southest Region of Turkey
}

\author{
Hadice Selimoglu Sen ${ }^{1}$, Ozlem Abakay ${ }^{1}$, Ayse Dalli ${ }^{2}$, Cengizhan Sezgi ${ }^{1}$, Mehmet Halis \\ Tanriverdi $^{3}$, Yilmaz Palanci ${ }^{4}$, Abdurrahman Abakay ${ }^{1 *}$ and Abdullah Cetin Tanrikulu ${ }^{1}$ \\ ${ }^{1}$ Department of Chest Disease, Medical Faculty, Dicle University, Diyarbakir, Turkey. \\ ${ }^{2}$ Department of Chest Disease, Education and Research Hospital, Diyarbakir, Turkey. \\ ${ }^{3}$ Department of Family Medicine, Medical Faculty, Dicle University, Diyarbakir, Turkey. \\ ${ }^{4}$ Department of Public Health, Medical Faculty, Dicle University, Diyarbakir, Turkey.
}

\begin{abstract}
Accepted 25 July, 2012
Drug resistance (DR) is an important problem in tuberculosis (TB) control programs. This study investigated the university hospital records of TB patients, along with the drug resistance ratios, the risk factors for developing multi drug resistance and their effects. Multi drug resistance is defined as the presence of both isoniazid $(H)$ and rifampicin $(R)$ resistance. The records of TB patients who had been diagnosed between January 2002 and December 2011 were evaluated retrospectively. Multi drug resistance was determined in 59 patients. The control group included 127 patients whose antibiograms had shown susceptibility to $H$, R, ethambutol (E) and streptomycin(S) (drug susceptible: DS) or who were resistant to one or more of these drugs, but not to both $\mathrm{H}$ and $\mathrm{R}$ simultaneously (mono resistant: MR). We randomly selected these 127 patients from 600 cases of TB that had been recorded through laboratory culture antibiogram data. There were no differences in age, gender, marital status or TB type between the multidrugresistant (MDR) and the control group, nor for the presence of cavities on their chest radiographies or smear positivity $(p<0.05)$. The study found a significantly increased risk of multi drug resistance among those with poor socioeconomic status, at 7.17 times higher (2.61-19.67), and those with a previous history of TB, at 5.61 times higher (2.10-15.07), as well as in patients who had diabetes mellitus, at 3.68 higher (1.15-11.79), in logistic regression analysis. Determining the risk factors for developing multi drug resistance in TB patients is critical in order to be able to prioritize the interventions to address this drug resistance. Although many risk factors for MDR TB have been reported, the most important appear to be a previous history of TB, previously administered TB treatment and inappropriate TB treatment.
\end{abstract}

Key words: Tuberculosis, multi drug resistance, risk factor.

\section{INTRODUCTION}

Tuberculosis (TB) continues to be a major health problem worldwide. Its high mortality and morbidity rates pose a serious threat both in our country and throughout the world. Providing adequate drug doses and duration of treatment and implementing appropriate combinations of regimens are very important in the struggle against TB.

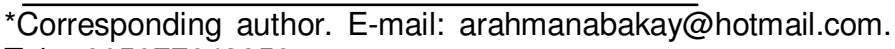
Tel: +905077643859 .
The adventure in successfully treating tuberculosis began in 1942 with the discovery of streptomycin, by Selman A. Waksman. Since that time, discoveries of new drugs have resulted in significant progress in the successful treatment of the disease. However, with the emergence of multi drug resistant and extensively drug resistant strains of TB, the treatment has become very difficult. World health organization (WHO)'s 2011 report on global TB control has provided a very important guide for the diagnosis, treatment and methods of combatting the disease. This report gives information about the burden 
of disease and death caused by TB and multi drug resistant (MDR) TB, as well as the treatment outcomes, financing mechanisms and new TB diagnostics, drugs and vaccines (WHO/HTM/TB/2011).

Drug resistance (DR) is an important problem in TB control programs and DR, TB rates are increasing globally. Multi drug resistance, which is defined as the presence of both isoniazid $(\mathrm{H})$ and rifampicin (R) resistance, is a growing health problem (Zignol et al., 2006). Although resistance to antituberculosis drugs first became apparent soon after the introduction of these drugs 50 years ago (Crofton, 1987), the development of multi drug treatment regimens succeeded in reducing the likelihood of resistance (Cohn, 1959). MDR TB first emerged in the 1990s when high mortality rates followed outbreaks in hospitals, in the United States. DR has often been the result of inappropriately or poorly implemented primary programs for the control of TB (Murray, 1998). Immigration and increased travel have also caused MDR strains of TB to spread from devoloping countries to developed countries. In 2001, there were an estimated 650,000 cases of MDR TB among the world's 12.0 million known cases of the disease (WHO/HTM/TB/2011).

Treatment for MDR is more complex, toxic and expensive, and also less effective, than treatment for TB that is susceptible to all drugs (Espinal et al., 2001), and is successful in only 60 to $70 \%$ of cases (Mitnick et al., 2003). Because of the current global economic crisis, the cost of MDR TB is particularly critical for many countries. For Turkey, which has moderate TB incidence, the disease remains a public health threat and an economic burden. According to Turkey's 2011 tuberculosis control report, based on data taken between 2005 and 2009, the country's MDR TB ratios ranged from 4.9 to $5.3 \%$ (Bozkurt, 2011). Some pilot studies in different parts of Turkey have shown high rates of both primary and secondary mono and multi drug resistant TB (Bengisun et al., 2000; Balci et al., 2000; Tahaoglu et al., 1994; Tanrikulu et al., 2008). However, there are few reports investigating the risk factors for MDR in Turkey (Tanrikulu et al., 2008; Karabay et al., 2004). This study was designed to investigate the risk factors for developing MDR TB in the city of Diyarbakir, in the Southeastern Anatolian Region of the country. The demographic details of the TB cases, including age, gender, marital status, radiological findings, TB types and smear positivity, were determined. The treatment results and ratios from our university hospital were evaulated.

\section{MATERIALS AND METHODS}

Turkey is composed of seven geographic regions. The socioeconomic status and level of development in the Southeastern Anatolian Region lags behind other regions. The city of Diyarbakir, which is located in this region, has one university hospital that serves approximately 10 million people, providing treatment and follow-up for the TB patients living in both the city center and surrounding towns. When peripheral hospitals suspect multi drug resistance in a TB patient, based on clinical, laboratory and radiological data, they send the samples to the Diyarbakır Dicle University's central laboratory, where culture and drug suspectibility tests can be performed using the BACTEC method.

This study used data from the central laboratory records of the university hospital. Because the study was retrospective, ethical approval was not required. All samples obtained from patients at the university hospital are routinely subjected to smear and culture processes, using the Loewenstein Jensen method. The hospital is able to do culture antibiograms for $H$, R, streptomycin (S) and ethambutol $(E)$, but not for pyrazinamide $(P)$.

The records of patients who had been diagnosed with TB between January 2002 and December 2011 were evaluated retrospectively. MDR TB was determined in 59 patients during this period. The control group included 127 patients whose antibiograms had shown susceptibility to $H, R, E$ and $S$ (drug susceptible: DS) or who were resistant to one or more of these drugs, but not resistant to both $\mathrm{H}$ and $\mathrm{R}$ simultaneously (mono resistant: MR). We randomly selected these 127 patients from 600 cases that had been recorded through laboratory culture antibiogram data. The demographic data that was investigated included gender, age, marital status, the presence of diabetes mellitus, a history of TB, the presence of cavities on posteroanterior (PA) chest radiography, the type of TB, smear and culture results, treatment results, previous TB treatment and drug resistance events, as well as socioeconomic status.

Drug susceptibility testing (DST) to first-line antibiotics was performed on at least one Mycobacterium tuberculosis isolate from each culture-positive patient $(H, R, E$ and $S$ for all isolates). The BACTEC radiometric system (Becton Dickinson, Sparks, MD, USA) was used to detect susceptibility to drugs, through standard techniques, in the university's central laboratory. The drug concentrations for identifying resistant status were $0.4 \mu \mathrm{g} / \mathrm{ml}$ for $\mathrm{H}$, $2.0 \mu \mathrm{g} / \mathrm{ml}$ for $\mathrm{R}, 4.0 \mu \mathrm{g} / \mathrm{ml}$ for $\mathrm{E}$ and $2.0 \mu \mathrm{g} / \mathrm{ml}$ for $\mathrm{S}$. The BACTEC method, which the central laboratory at Dicle University has used since 2000, has been demonstrated to be $100 \%$ sensitive in identifying resistance to first-line drugs (Balcı et al., 2000).

The resistance pattern was classified as either primary or secondary in patients with MDR TB. Primary resistance, which is the resistance belonging to the source from which the bacillus was transmitted, is determined in new tuberculosis patients who have not yet used TB drugs or have used drugs for less than one month. Primary resistance is parallel to the rate of secondary resistance in a population. Secondary resistance results from increased resistant mutants following inappropriate treatment, resulting from drug choice, early discontinuation of drugs and lack of compliance with treatment, in TB patients previously infected with susceptible bacilli. Increased secondary resistance is a marker of inadequate TB treatment (Durmaz, 2003).

Resistance has been defined according to WHO guidelines, and MDR TB is defined as resistant to two first-line drugs: $H$ and $R$. Data were recorded on standard forms that had been previously prepared.

\section{Definitions of treatment outcomes (WHO/HTM/TB/2011)}

Cured: A patient who was initially smear-positive and who was smear-negative in the last month of treatment and on at least one previous occasion.

Completed treatment: A patient who completed treatment but did not meet the criteria for cure or failure. This definition applies to pulmonary smear-positive and smear-negative patients and to patients with extrapulmonary disease.

Died: A patient who died from any cause during treatment.

Failed: A patient who was initially smear-positive and who remained smear-positive at month five or later during treatment. 
Table 1. Demographic data for patients.

\begin{tabular}{lcccc}
\hline \multirow{2}{*}{ Data for patients } & $\mathbf{n}$ & MDR + & \multicolumn{2}{c}{ DS or MR } \\
\cline { 2 - 5 } Marital status & 20 & 33.9 & $\mathbf{n}$ & $\%$ \\
Single & 39 & 66.1 & 33 & 26.0 \\
Married & 59 & 100 & 127 & 74.0 \\
Total & & & & 100 \\
Cavite & 25 & 42.3 & 60 & \\
Absent & 34 & 57.7 & 66 & 47.6 \\
Present & 59 & 100 & 126 & 52.4 \\
Total & & & & 100 \\
& & & & \\
Type of TB & 50 & 84.7 & 105 & 82.7 \\
Pulmonary TB & 9 & 15.3 & 22 & 17.3 \\
Extrapulmonary TB & 59 & 100 & 127 & 100 \\
Total & & & & \\
& & & & \\
Smear positivity & 46 & 77.9 & 44 & 65.3 \\
Positive & 13 & 22.0 & 127 & 100 \\
Negative & 59 & 100 & & \\
Total & & & & \\
\hline
\end{tabular}

MDR: multi drug resistant, DS: drug susceptible, MR: mono resistant, TB: Tuberculosis.

Defaulted: A patient whose treatment was interrupted for two consecutive months or more.

Not evaluated: A patient whose treatment outcome is not known.

Successfully treated: A patient who was cured or who completed treatment.Inappropriate treatment was defined as lack of compliance with the therapeutic regimens recommended in guidelines and with the recommended duration; also defined as adding or excluding drugs during treatment (WHO/HTM/TB/2011).

The presence of social health insurance was used to investigate the effects of social and economic parameters in the development of multi drug resistance. Because the social health insurance inTurkey is linked to having a regular job and an adequate level of income, patients who had no social health insurance were classified as low socioeconomic status, while patients with insurance were considered as having high socioeconomical status.

\section{Statistical analyses}

All of the data were recorded and analyzed by SPSS 15.0 for Windows (SPSS Inc., Chicago, IL, USA). Categorical variables were analyzed with the chi-square test; numerical variables were analyzed using the Student t test. The predictors of MDR were investigated with multivariable analysis, using the logistic regression method, and $p<0.05$ was considered significant.

\section{RESULTS}

The study included 59 patients with MDR and 127 patients with DS or MR TB as the control group. The mean age was $38.58 \pm 18.24(34.55 \pm 16.73$ in the MDR group and $40.23 \pm 18.94$ in the control group), with the range from 12 to 86 . In terms of the genders of the patients, 113 (36 in the MDR group) were male and 73 (23 in MDR group) were female. Among the patients with MDR, 25 (42.4\%) were recorded as having primary drug resistance, with $34(57.6 \%)$ having secondary drug resistance. There was no difference between the MDR group and the controls for age, gender, marital status, TB type, the presence of cavity on the chest radiography and smear positivity $(p<0.05)$. Table 1 shows the demographic data of the patients.

No patients in the MDR group were provided with treatment under direct observation. Treatment results are given in Table 2. The following risk factors for MDR were all found to be significant when evaluated with univariate analysis methods: poor socioeconomic status, previous history of TB, previously administered TB treatment, inappropriate TB treatment, diabetes mellitus and noncompliance with TB treatment. When these risk factors were analyzed with logistic regression analysis, the MDR risk was significantly increased, regardless of any other risk factors, rising by 7.02 for poor socioeconomic status, by 5.50 for previous TB history and by 3.66 in patients with diabetes mellitus (Table 3 ).

\section{DISCUSSION}

The tuberculosis treatment process is long and difficult for patients and health workers. The high number of 
Table 2. Treatment results of all cases.

\begin{tabular}{lcccc}
\hline \multirow{2}{*}{ Treatment result } & \multicolumn{2}{c}{ MDR } & \multicolumn{2}{c}{ DS and MR } \\
\cline { 2 - 5 } & $\mathbf{n}$ & $\%$ & $\mathbf{n}$ & $\%$ \\
\hline Treatment completed & 41 & 69.5 & 95 & 75.8 \\
Cure & 7 & 11.9 & 8 & 6.3 \\
Continued treatment & 10 & 16.9 & 18 & 14.2 \\
Treatment abandoned & 1 & 1.7 & 6 & 4.7 \\
Total & 59 & 100 & 127 & 100 \\
\hline
\end{tabular}

MDR: multi drug resistant, DS: drug susceptible, MR: monoresistant.

Table 3. Analyze results risk factors of MDR with logistic regresyon.

\begin{tabular}{|c|c|c|c|c|}
\hline Variables & $\mathbf{n}$ & MDR (\%) & Odds ratio $(95 \% \mathrm{Cl})$ & Adjusted odds ratio $(95 \% \mathrm{Cl})$ \\
\hline \multicolumn{5}{|l|}{ Age } \\
\hline$\geq 40$ years & 76 & 22.4 & $1^{a}$ & $1^{\mathrm{ns}}$ \\
\hline$\leq 39$ years & 110 & 38 & $2.14(1.11-4.16)$ & $1.71(0.74-3.96)$ \\
\hline \multicolumn{5}{|l|}{ DM } \\
\hline Absent & 166 & 28.9 & $1^{a}$ & $1^{a}$ \\
\hline Present & 20 & 55.0 & $3.00(1.17-7.71)$ & $3.68(1.15-11.79)$ \\
\hline \multicolumn{5}{|c|}{ TB history (the patient's) } \\
\hline Absent & 131 & 18.3 & $1^{\mathrm{c}}$ & $1^{\mathrm{c}}$ \\
\hline Present & 55 & 63.6 & $7.80(3.85-15.80)$ & $5.50(2.05-14.76)$ \\
\hline \multicolumn{5}{|c|}{ Socioeconomic statements } \\
\hline Bad & 30 & 66.7 & $1^{\mathrm{c}}$ & $1^{\mathrm{c}}$ \\
\hline Good & 156 & 25.0 & $6.00(2.59-13.91)$ & $7.17(2.61-19.67)$ \\
\hline \multicolumn{5}{|c|}{ The living space } \\
\hline Village- Town & 64 & 17.2 & $1^{\mathrm{b}}$ & $1^{\mathrm{a}}$ \\
\hline Country & 122 & 39.3 & $3.13(1.49-6.58)$ & $2.79(1.15-6.76)$ \\
\hline \multicolumn{5}{|c|}{ Inappropriate treatment } \\
\hline Absent & 156 & 25.6 & $1^{c}$ & $1^{\text {ns }}$ \\
\hline Present & 30 & 63.3 & $5.01(2.20-11.43)$ & $0.93(0.28-3.06)$ \\
\hline
\end{tabular}

a: $p<0.05, b: p<0.01, c: p<0.001$, ns: not significant $C l$ : confidence interval, TB: tuberculosis, DM: diabetes mellitus.

medications involved, the necessity for regular drug use and the side effects of the drugs are just some of these challenges. Drug resistance is a serious problem that can affect the success of treatment. Drug resistant TB is an artifact constitution arising from poor TB control programs, prescriptions for inappropriate treatment regimens, a lack of access to laboratory culture antibiograms, mismatched treatment and also poor quality drug supplies. These have all contributed to the emergence of drug resistance and led to an increase in the disease. Inappropriate treatment of the TB and patient noncompliance with their treatments have been reported as additional factors contributing to the increases in drug resistance (Karabay, 2004).

Treatment failure is reported as the strongest factor associated with multi drug resistance, followed by exposure to an MDR TB case or to a case that has failed in treatment (Velasquez et al., 2008; Caminero, 2008). Determining multi drug resistance in patients is considered as a marker of inappropriate TB prevention and control measures in the countries where primary drug resistance is high. It is also seen as a sign that inefficient therapeutic protocol has been implemented for tuberculosis in countries where secondary drug 
resistance is high (Durmaz et al., 2003).

The incidence of MDR TB has been estimated to be up to 10 times higher after unsuccessful treatment (PabloMendez et al., 1998), and the risk for multi drug resistance has been found to be 10.23 times higher in patients who had previously taken TB medication than among patients who had not (Faustini et al., 2006). Previously administered TB treatment is an important risk factor for the development of drug resistance, and it has been reported that this situation has increased the development of drug resistance by five times, on average (Lomtadze et al., 2009). According to another report, the history of previous TB treatment and of irregular treatment for more than three months also increases the risk of multi drug resistance (Mendoza et al., 1997). In addition, the risk of multi drug resistance in people who had previously been given TB treatment was found to be 10.54 times higher than in those who had not received treatment (Kliiman and Altraja, 2009). A study performed in our region also found previous treatment to be a risk factor for MDR TB (Tanrikulu et al., 2008).

The highest MDR TB rates among isolates were reported from Nepal (48\%), Gujarat, in India (34\%), New York (30\%), and Bolivia and Korea (15\% each), based on a wide-ranging review (Cohn et al., 1997). The total combined resistance rate to one or more drugs was found to be $40.2 \%$ in a previous study from our region (Balcl et al., 2000). The studies reported that, in Turkey, primary drug resistance ranges from 1 to $5 \%$ and secondary drug resistance varies between 4 and $17 \%$ (Durmaz, 2005).

When we evaluated the risk factors for multi drug resistance with univariate analysis methods, we found that poor socioeconomic status, previous TB history, previously administered TB treatment, inappropriate TB treatment, diabetes mellitus and noncompliance with TB treatment were significant risk factors. In addition, a previous history of TB increased the risk of multi drug resistance by 5.5 times in multiple analysis. A relationship between the previous TB exposure history of the patient and drug resistance was reported in another study as well (Moniruzzaman et al., 2006).

Previous TB exposure was found to be an important risk factor that increased MDR TB risk in this study also. Although directly observed treatment has not been implemented for patients with MDR TB in Diyarbakir, this type of treatment has been used for the patients without MDR TB since the end of 2006. The significant effect of noncompliance to TB treatment in terms of the development of multi drug resistance was determined through statistical analysis in our study. This result suggests that treatment with direct observation should be given to patients with MDR TB.

MDR TB, as well as lung cavities, has been associated with female patients in some settings (Lomtadze et al., 2009; Barroso et al., 2003). However, another earlier study found no relationship between gender, the presence of diabetes mellitus, a history of close contact with TB or MDR TB (Suárez-García et al., 2009). In our study, gender and a history of close contact with TB were not identified as risk factors for multi drug resistance. However, the presence of DM was found to be associated with a high MDR TB risk.

In another study, no relationship was found between educational status, gender and drug resistance (Moniruzzaman et al., 2006), and a previous study has reported that $56.7 \%$ of MDR TB cases occurred among those with poor economic status (Wahab et al., 2009). Our study determined that low socioeconomic status made the risk of MDR 7.02 times higher. Low socioeconomic status was also considered to be an obstacle to appropriate and necessary treatment once the initial diagnosis was made.

\section{Conclusions}

In conclusion, determining the risk factors for developing and transmitting MDR TB is important in order to be able to prioritize interventions to address drug resistance. Among the many risk factors for MDR TB that have been reported, the most critical appear to be a previous history of TB, previously administered TB treatment and inappropriate TB treatment. According to this study, socioeconomic factors and the presence of DM also play a role in this. Health workers need to be able to reach all TB patients and to give treatment under direct observation, to minimize the effects of a patient's risk factors for the development of drug resistance. Patients with new diagnoses of TB require close monitoring in order to prevent MDR.

Infection control programs, early diagnosis, appropriate management of the cases and addressing the high default rates that result from both sensitive and resistant TB are important factors for reducing transmission of MDR TB. Isolates of MDR TB must be submitted to the reference laboratory for confirmation. Furthermore, this requirement should also be supported and applied by national health policies and control programs. We believe that, if all of these measures are taken, MDR TB rates may fall significantly.

\section{REFERENCES}

Balci I, Dikensoy O, Bayram A, Filiz A (2000). Drug-resistant tuberculosis at the University Hospital in Gaziantep, South-Eastern Turkey. J. Int. Med. Res. 28:300-306.

Barroso EC, Salani RM, Oliveira R, Oliveira AL, Brasileiro B, Jorge Luís NR (2003) Risk factors for acquired multidrug-resistant tuberculosis. J Pneumologia 29(2).

Bengisun JS, Karnak D, Palabiyikoglu I, Saygun N (2000). Mycobacterium tuberculosis drug resistance in Turkey, 1976-97. Scand J. Infect. Dis. 32(5):507-510.

Bozkurt H (2011). Turkey Tuberculosis Control Report. Ankara: Fersa Offset, pp 47-74.

Caminero JA (2008). Likelihood of generating MDR-TB and XDR-TB 
under adequate National Tuberculosis Control Programme implementation. Int. J. Tuberc. Lung Dis. 12(8):869-877.

Cohn DL, Bustreo F, Raviglione MC (1997). Drug-resistant tuberculosis: review of the worldwide situation and the WHO/IUATLD Global Surveillance Project. Clin Infect Dis. 24 (1): S121.

Cohn ML, Middlebrook G, Russell WF Jr (1959). Combined drug treatment of tuberculosis.I. Prevention of emergence of mutant populations of tubercle baciili resistant to both streptomycin and isoniazid in vitro. J. Clin. Invest. 38:1349-1355.

Crofton J (1987). The prevention and management of drug-resistant tuberculosis. Bull. Int. Union Tuberc. Lung Dis. 62 (1-2):6-11.

Durmaz R, Ozerol IH, Durmaz B, Gunal S, Senoglu A (2003). Evliyaoglu E. Primary drug resistance and molecular epidemiology of Mycobacterium tuberculosis isolates from patients in a population with high tuberculosis incidence in Turkey. Microb. Drug Resist. 9(4):361-366.

Durmaz R (2005). The Resistance Problem in Mycobacterium tuberculosis. Joint Dis. Rel. Surg. 19(2):107-110.

Espinal MA, Laszlo A, Simonsen L, Boulahbal F, Kim SJ, Reniero A Hoffner S, Rieder HL, Binkin N, Dye C, Williams R, Raviglione MC (2001). Global trends in resistance to antituberculosis drugs. World Health Organization-International Union against Tuberculosis and Lung Disease Working Group on Anti-Tuberculosis Drug Resistance Surveillance. N. Engl. J. Med. 344(17):1294-303.

Faustini A, Hall A J, Perucci CA (2006). Risk factors for multidrug resistant tuberculosis in Europe: a systematic review. Thorax 61:158163.

Global tuberculosis control (2011). Geneva: World Health Organization; 2011 (WHO/HTM/ TB/2011).

Karabay O, Otkun M, Akata F, Karlikaya C, Tuğrul M, Dündar V (2004). Antituberculosis drug resistance and associated risk factors in the European section of Turkey. Indian J. Chest. Dis. Allied Sci. 46(3):171-177.

Kliiman K, Altraja A (2009). Predictors of extensively drug-resistant pulmonary tuberculosis. Ann. Int. Med. 150(11):766-775.

Lomtadze N, Aspindzelashvili R, Janjgava M, Mirtskhulava V, Wright A, Blumberg HM, Salakaia A (2009). Prevalence and risk factors for multidrug-resistant tuberculosis in the Republic of Georgia: a population-based study. Int. J. Tuberc. Lung. Dis. 13(1):68-73

Mendoza MT, Gonzaga AJ, Roa C (1997). Nature of drug resistance and predictors of multidrug-resistant tuberculosis among patients seen at the Philippine General Hospital, Manila, Philippines. Int. J. Tuberc. Lung Dis. 1:59-63.

Mitnick C, Bayona J, Palacios E (2003). Community-based therapy for multidrug resistant tuberculosis in Lima, Peru. N. Engl. J. Med. 348:119-127.

Moniruzzaman A, Elwood RK, Schulzer M, FitzGerald JM (2006). A population-based study of risk factors for drug-resistant TB in British Columbia. Int. J. Tuberc. Lung. Dis. 10(6):631-8.
Murray JF (1998). Tuberculosis and HIV infection: a global perspective. Respiration 65:335-342.

Pablo-Mendez A, Raviglione MC, Laszlo A (1998). Global surveillance for antituberculosis-drug resistance, 1994-1997. N. Engl. J. Med. 338:1641-9.B/2011.6).

Tahaoglu K, Kizkin O, Karagoz T (1994). High initial and acquired drug resistance in pulmonary tuberculosis in Turkey. Tuber. Lung. Dis. 75:324-328.

Tanrikulu AC, Hosoglu S, Ozekinci T, Abakay A, Gurkan F (2008). Risk factors for drug resistant tuberculosis in southeast Turkey. Trop. Doct. 38(2):91-93.

Velasquez GE, Yagui M, Cegielski P, Asencios L, Bayona J (2011). Targeted drug-resistance testing strategy for multidrug-resistant tuberculosis detection, Lima, Peru, 2005-2008. Emerg. Infect. Dis. 17(3): 432-440.

Wahab F, Ashraf S, Khan N, Anwar R, Afridi MZ (2009). Risk factors for multi-drug resistant tuberculosis in patients at tertiary care hospital, Peshawar. J. Coll. Physicians Surg. Pak. 19(3):162-164.

Zignol M, Hosseini MS, Wright A, Weezenbeek CL, Nunn P, Watt CJ, Williams BG, Dye C (2006). Global incidence of multidrug-resistant, tuberculosis. J. Infect. Dis. 194(4):479-485. 